\title{
An Alternative to Wave Mechanics on Curved Spaces
}

\author{
Roman Tomaschitz ${ }^{1}$ \\ Received April 24, 1991
}

\begin{abstract}
Geodesic motion in infinite spaces of constant negative curvature provides for the first time an example where a basically quantum mechanical quantity, a ground-state energy, is derived from Newtonian mechanics in a rigorous, nonsemiclassical way. The ground state energy emerges as the Hausdorff dimension of a quasi-self-similar curve at infinity of three-dimensional hyperbolic space $\mathrm{H}^{3}$ in which our manifolds are embedded and where their universal covers are realized. This curve is just the locus of the limit set $\Lambda(\Gamma)$ of the Kleinian group $\Gamma$ of covering transformations, which determines the bounded trajectories in the manifold; all of them lie in the quotient $C(\Lambda) \backslash \Gamma, C(\Lambda)$ being the hyperbolic convex hull of $\Lambda(\Gamma)$. The three-dimensional hyperbolic manifolds we construct can be visualized as thickened surfaces, topological products $I \times S, I$ a finite open interval, the fibers $S$ compact Riemann surfaces. We give a short derivation of the Patterson formula connecting the ground-state energy with the Hausdorff dimension $\delta$ of $\Lambda$, and give various examples for the calculation of $\delta$ from the tessellations of the boundary of $H^{3}$, induced by the universal coverings of the manifolds.
\end{abstract}

\section{INTRODUCTION}

We consider three-dimensional Riemannian spaces of infinite volume and constant negative curvature $-1 / R^{2}$. A typical class of such manifolds is that of smooth thickened surfaces: imagine a sphere with some handles attached, and imagine the material of which this surface is formed as thick. One gets a three-dimensional space with an interior and exterior boundary, topologically $I \times S$, fibering over a finite interval $I$, the fibers being compact Riemann surfaces of genus $g \geq 2$. If we take $I$ open, i.e., if we remove the boundary, we can endow these manifolds with a metric of constant negative curvature that gives rise to infinite non-Euclidean volume.

\footnotetext{
${ }^{1}$ Instituts Internationaux de Physique et de Chimie Solvay, Université Libre de Bruxelles, B-1050 Bruxelles, Belgium.
} 
We will be concerned with the geometric shapes of the classical geodesic trajectories. There are bounded trajectories lying during their whole time evolution between $t=-\infty$ and $t=+\infty$ (our spaces are geodesically complete) in a non-Euclidean sphere of finite radius, and unbounded ones that tend either for $t \rightarrow+\infty$ or $t \rightarrow-\infty$ to the boundary. In fact, the bounded trajectories occupy a three-dimensional, finite, convex domain $C(\Lambda) \backslash \Gamma$; almost all of them lie dense in it. There exist countably many homotopy classes of closed loops, too, that are of crucial importance if one tries to get "classical information" about excited states via Selberg's trace formula (Hejhal, 1976; Hurt, 1983: McKean, 1972), but the trace will not be dealt with here. Bounded trajectories are more the exception than unbounded ones; in the sense of Riemann-Lebesgue measure on the sphere at infinity of hyperbolic space (cf. Section 1), almost all are unbounded. Nevertheless, there are enough to provide a square-integrable ground state for the Schrödinger operator, which is in general well separated from the continuous spectrum.

The clue that relates the ground-state eigenvalue of the Schrödinger operator on the manifold with the bounded trajectories is uniformization: taking $H^{3}$ as a model for the universal covering space (cf. Section 1), we realize the group of covering transformations as a Kleinian group $\Gamma$ of Möbius transformations acting on $H^{3}$. We construct a concrete realization of the manifold in $H^{3}$ as a fundamental polyhedron $F$ for $\Gamma$. The group $\Gamma$ applied to $F$ gives a polyhedral tiling of hyperbolic space. Accumulation points of tiles on the boundary of $H^{3}$ constitute the fractal quasi-self-similar limit set $\Lambda$ of $\Gamma$ (Apanasov, 1991; Krushkal et al., 1986; Maskit, 1974; Sullivan, 1982). The quotient $C(\Lambda) \backslash \Gamma$ of the hyperbolic convex hull $C(\Lambda)$ of $\Lambda$ (Epstein and Marden, 1987), which contains the bounded trajectories, is just the intersection of $C(\Lambda)$ and $F$ with the face-identification of $F$ imposed. In fact, $\Lambda$ is the set of initial and end points of lifts of bounded trajectories into the universal cover (Tomaschitz, 1991, 1992a,b).

On the other hand, the ground-state energy (cf. Section 2) of the Schrödinger operator of quantized geodesic motion is connected with the Hausdorff dimension $\delta$ of $\Lambda$ via the formula $E_{0}=\left(-\hbar^{2} / 2 R^{2} m\right)(\delta-1)^{2}$ (Patterson, 1975, 1976, 1987; Phillips and Sarnak, 1985; Sullivan, 1979). This formula provides a simple example for the reconstruction of a quantum mechanical quantity, a ground-state energy, from classical mechanics, namely the set of bounded trajectories.

In Section 1 and the Appendix we discuss briefly normal forms of spaces of constant curvature, i.e., fundamental polyhedra of Kleinian groups in hyperbolic space, their deformations, and the tessellations of their universal cover. In Section 2 we discuss some aspects of classical mechanics, bounded trajectories, convex hulls of limit sets, and the Schrödinger equation in these 
spaces, and sketch the derivation of the Patterson formula connecting energy and Hausdorff dimension. Section 3 is devoted to numerical examples: generation of tessellations, their characteristic curves, their limit sets, and the calculation of the Hausdorff dimension.

\section{NORMAL FORMS OF SPACES OF CONSTANT CURVATURE: FUNDAMENTAL POLYHEDRA}

Three-dimensional spaces of constant curvature $-1 / R^{2}$ and their universal covers are modeled on the upper shell of the Minkowski hyperboloid $x_{0}^{2}-x_{1}^{2}-x_{2}^{2}-x_{3}^{2}=-R^{2}$, endowed with the Minkowski metric $d s^{2}=d x_{0}^{2}-d \mathbf{x}^{2}$. The isometry group of this space is the Lorentz group $\mathrm{SO}^{+}(3,1)(+$ means here the subgroup that preserves the upper shell of the hyperboloid). We will use other isometric versions of hyperbolic space, the Poincare ball $B^{3}$ and the half-space $\mathrm{H}^{3}$; we sketch them briefly here for self-containedness; for details see Ahlfors (1981), Beardon (1983), and Milnor (1982).

$B^{3}$ is an open three-dimensional ball $|\mathbf{x}|<R$, endowed with $d s^{2}=$ $4\left(1-|\mathbf{x}|^{2} / R^{2}\right)^{-2} d \mathbf{x}^{2} ; H^{3}$ is the upper half-space of $\mathbb{R}^{3}, y_{3}>0$, with $d s^{2}=$ $R^{2} y_{3}^{-2} d y^{2}$. In both spaces the conformal factor of the metric gets infinite at the boundary, the sphere $S_{\infty}$ or plane $\mathbb{C}$ (compactified) at infinity. The geodesics in this geometry are in the $H^{3}$ model semicircles orthogonal to the complex plane, and the totally geodesic planes are hemispheres resting on the plane at infinity; in $B^{3}$ geodesics are arcs of circles orthogonal to $S_{\infty}$, and the geodesic planes are caps of spheres orthogonal to $S_{\infty}$.

The action of the Lorentz group on $\mathrm{H}^{3}$ is realized as follows: $\mathrm{SO}^{+}(3,1)$ is isomorphic to $S L(2, \mathbb{C}) /\{ \pm 1\}$, the group of Möbius transformations in the complex plane: $z \rightarrow(a z+b) /(c z+d) ;\left(\begin{array}{c}a b \\ c d\end{array}\right) \in S L(2, \mathbb{C})$. Decomposing $z \rightarrow(a z+b) /(c z+d)$ in four successive transformations $z \rightarrow z+d / c, z \rightarrow c^{2} z$, $z \rightarrow-1 / z, z \rightarrow z+a / c$, we see that it consists of reflections in circles or straight lines. The group action on $\mathbb{C}$ is now lifted to $H^{3}$ by reflecting points of $H^{3}$ in hemispheres with these circles as base circles (Beardon, 1983). (A plane orthogonal to $\mathbb{C}$ also is a totally geodesic plane in $H^{3}$.) The analytic formula for the group action in $H^{3}$ can easily be read off from the above decomposition:

$$
\left(z, y_{3}\right) \rightarrow\left(\frac{(a z+b) \overline{(c z+d)}+a \bar{c} y_{3}^{2}}{|c z+d|^{2}+|c|^{2} y_{3}^{2}}, \frac{y_{3}}{|c z+d|^{2}+|c|^{2} y_{3}^{2}}\right)
$$

where we used $z=y_{1}+i y_{2}$. The group action in $B^{3}$ is more complicated (Ahlfors, 1981). 
Hyperbolic manifolds are now constructed by identifying the faces of a non-Euclidean polyhedron $F$ in $H^{3}$ with elements of the Lorentz group. First we realize the identification pattern of the base circles (e.g., Figure 2 or Figure 5) of the hemispheres on which the faces of the polyhedron lie in the complex plane. If we identify, as indicated in the figures, the boundary arcs $P_{i} P_{i+1}$ of domain $f_{1}$, we get a surface of genus 5 (Figure 2) or 6 (Figure 5), i.e., topologically a sphere with 5 (6) handles attached. Likewise, if we perform the same identification of the sides $Q_{i} Q_{i+1}$, the boundary of domain $f_{2}$ that comprises the point at infinity of $\mathbb{C}$, we get again a surface of genus 5 (6). These two surfaces lying at infinity of hyperbolic space are the two boundary components of a three-dimensional manifold which is obtained by identifying the hemispheres with the lifted transformations. The domain lying above these hemispheres and $f_{1}$ and $f_{2}$ is a non-Euclidean polyhedron $F$ with identified faces on the hemispheres and two free faces $f_{1}, f_{2}$. The identification gives a thickened surface of genus $5(6)$ with $f_{1}$ and $f_{2}$ as exterior and interior boundaries (Figure 1), where the metric gets singular. Therefore, a particle cannot cross in a finite time from $f_{1}$ to $f_{2} ;$ see (2.2). (In the $B^{3}$ model the ring of base circles lies on $S_{\infty}$, and $F$ is realized by placing spherical caps orthogonal to $S_{\infty}$ onto them.)

If we apply $\Gamma$, the group generated by the lifted transformations, to $F$, a tiling of $H^{3}$ results; the images $\Gamma(F)$ tessellate the interior of the hemispheres, which is just the condition that the metric of $H^{3}$ fits smoothly on the identified faces of $F$, inducing globally a metric of constant negative curvature on the manifold. $H^{3}$ appears as the universal covering space with $\Gamma$ as the group of deck transformations that uniformizes the manifold, emerging as the quotient $H^{3} \backslash \Gamma$. In order that the $\Gamma(F)$ constitute really a tessellation of $H^{3}$ without holes and overlapping, the base circles have to fulfill certain relations among their centers and radii; cf. the Appendix. Only $12(g-1)$ real parameters ( $g$ is the genus of the fibers) of the circles $\left(M_{i}, r_{i}\right)$ can be varied independently [deformation space (Bers, 1970)], giving rise to fundamental polyhedra that represent nonisometric manifolds, i.e., two polyhedra cannot be mapped onto each other by a lifted Möbius transformation that respects the identification of the faces.

Figures 3, 4, 6, and 8 show tessellations of the complex plane induced by tilings $\Gamma(F)$ of $H^{3}$. They are obtained by applying $\Gamma$ to $f_{1}$ and $f_{2}$, the free faces of $F$. The fractal curve in the figures comprises just the accumulation points of the images $\Gamma(F)$ of the $H^{3}$-tessellation, the limit set $\Lambda(\Gamma)$ (cf., e.g. Lehner, 1964). In the plane at infinity of $H^{3}, \Gamma\left(f_{1}\right)$ tessellates the interior (Figures 7 and 9) and $f_{2}$ the exterior of this Jordan curve. Its Hausdorff dimension determines the ground state of the Laplace-Beltrami operator, the Schrödinger operator for geodesic motion. 


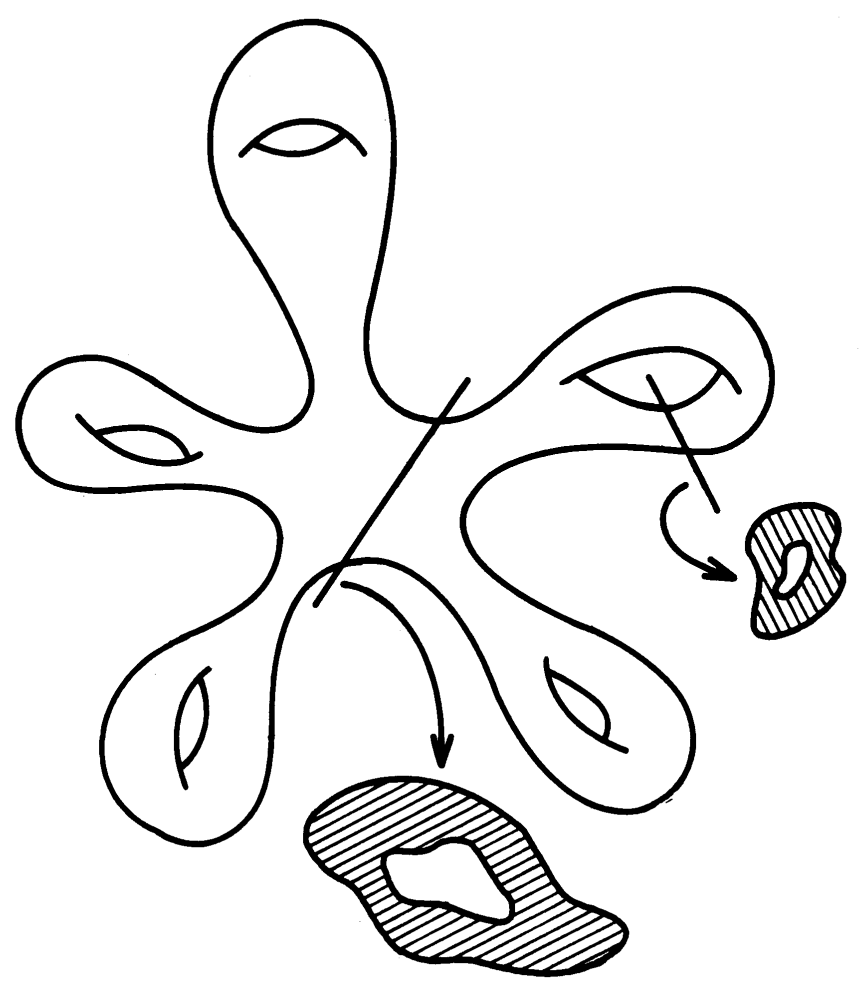

Fig. 1. Topological structure of the hyperbolic spaces corresponding to the identification pattern in Figure 2: thickened surfaces of genus five, topological products of a finite interval and a compact Riemann surface. The metric gets singular at the interior and exterior boundaries, giving rise to infinite hyperbolic volume.

\section{CLASSICAL MECHANICS, CONVEX HULLS OF TRAJECTORIES, AND THE GEOMETRIC NATURE OF THE QUANTUM MECHANICAL GROUND-STATE ENERGY}

Geodesic motion in hyperbolic spaces is realized by projecting geodesics of the covering space $H^{3}$ (or $B^{3}$, respectively) into the manifold. Every arc of the semicircle that lies in an image $\gamma(F), \gamma \in \Gamma$, is projected back via $\gamma^{-1}$ into $F$, the fundamental polyhedron. One can view the image of this covering projection as the intersection of $F$ with all the $\Gamma$-images of the $H^{3}$ (or $B^{3}$ )geodesic. The face-identification of $F$ also gives the identification of the projected arcs, so that we get a smooth curve in the manifold. A particle moving with velocity $\mathbf{v}:=d \mathbf{s} / d t=2\left(1-|\mathbf{x}|^{2} / R^{2}\right)^{-1} d \mathbf{x} / d t$ along this curve 


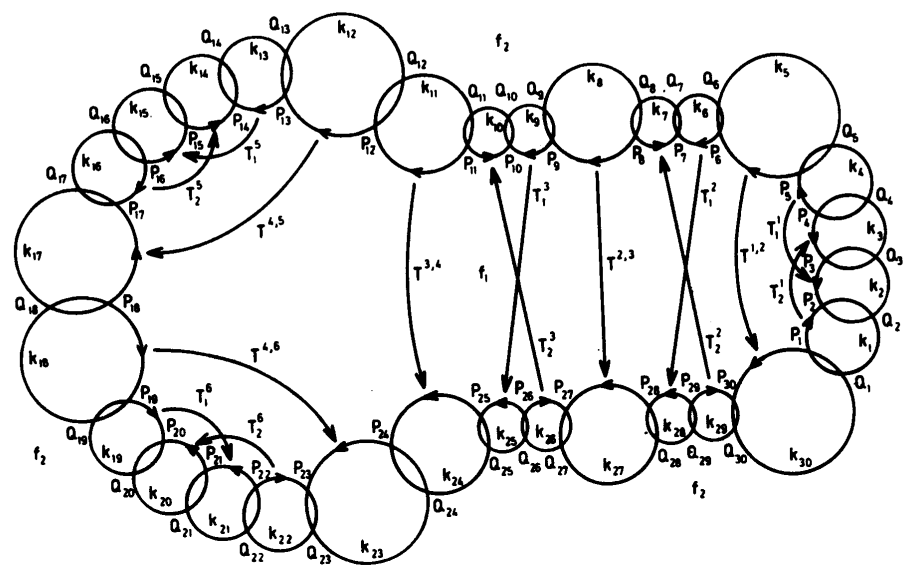

Fig. 2. Identification of the sides of the interior polygon $\left(P_{i}\right.$-vertices) gives a surface of genus five, likewise the identification of the exterior polygon ( $Q_{i}$-vertices) in the extended complex plane: the interior and exterior boundaries of the manifolds; see also the caption of Figure 5.

satisfies Newton's equations

$$
\frac{d \mathbf{v}}{d t}=\frac{1}{R^{2}}\left[\mathbf{x} \cdot \mathbf{v}^{2}-\mathbf{v} \cdot(\mathbf{x} \cdot \mathbf{v})\right]
$$

derived from the $B^{3}$-metric $d s^{2}=4\left(1-|\mathbf{x}|^{2} / R^{2}\right)^{-2} d \mathbf{x}^{2}$ induced on $F$. We see immediately that $|\mathbf{v}|$ is a constant of motion.

From (2.1) we calculate the time $t-t_{0}$ that a particle needs to move from point $\mathbf{x}_{0}$ to $\mathbf{x}$ :

$$
\sinh ^{2}\left(\frac{1}{2} \frac{|\mathbf{v}|}{R}\left(t-t_{0}\right)\right)=\frac{1}{R^{2}} \frac{\left|\mathbf{x}-\mathbf{x}_{0}\right|^{2}}{\left(1-|\mathbf{x}|^{2} / R^{2}\right)\left(1-\left|\mathbf{x}_{0}\right|^{2} / R^{2}\right)}
$$

It can never reach the boundary in a finite time. Increasing the curvature radius, $R \rightarrow \infty$, we recover motion in flat space. (Note that $d s^{2} \rightarrow 4 d \mathbf{x}^{2}$; we have to rescale lengths by $1 / 2$.)

If the end points of the semicircle that is projected do not lie in the limit set $\Lambda(\Gamma)$, it intersects only finitely many polyhedra of the tessellation, and its projection consists of finitely many arcs, the first and the last touching for $t= \pm \infty$ the plane at infinity: we get an unbounded trajectory.

Bounded trajectories are obtained by projections of semicircles which have both initial and end point in the limit set. Such a semicircle intersects infinitely many $\Gamma$-images of $F$. The image of its projection consists of infinitely many arcs, or accidentally finitely many which give rise to closed loops. All these arcs are uniformly separated from the plane at infinity by a finite Euclidean minimum distance. Note first that $\Gamma$ leaves the limit set invariant. 
Fig. 3. The faces of the polyhedra of the $H^{3}$-tessellation lie on hemispheres placed on the circles. Every polyhedron also has two free faces at infinity, one in the interior, the other in the exterior of the Jordan curve $(\delta=1.323)$, which are bounded by the same circles. The base circles and face-identifying mappings of the fundamental polyhedron $F$ from which the tessellation originates (Section 3 ) are depicted in Figures 13 and 2. With this identification $F$ is topologically a thickened surface of genus five that inherits the metric of $H^{3}$.

Therefore $\Gamma$ maps semicircles with initial and end point in $\Lambda(\Gamma)$ again onto such semicircles. The complete image of a projection is just the intersection of $F$ with all the $\Gamma$-images of the semicircle. Thus, it consists of arcs which lie on circles that intersect the hemispheres and have initial and end point in $\Lambda(\Gamma)$. Because $\Lambda(\Gamma)$ has a finite minimum separation from the boundary of $f_{1}$ and $f_{2}$, which is determined by the base circles of the hemispheres (see Figures 2-4) and Figures 5, 6, and 8), it follows that the arcs lying above the hemispheres have a common finite minimum separation from the plane at infinity. The trajectory which they constitute is therefore bounded. 
Fig. 4. The fundamental polyhedron of this tessellation is a continuous deformation of that in Figure 3; the induced metric gives again a manifold whose fibers are surfaces of genus five, but which is nonisometric to that in Figure 3. The Hausdorff dimension $\delta$ of the Jordan curve, the set of accumulation points of the tiling, gives the ground-state energy of the Schrödinger operator on the manifold. $\delta=1.307$.

We sketch briefly a picture of the hyperbolic convex hull $C(\Lambda)$ of $\Lambda(\Gamma)$, whose quotient $C(\Lambda) \backslash \Gamma$ is the domain in which all bounded trajectories lie. $C(\Lambda)$ is, analogous to the Euclidean case, the closure of the common intersection of all hyperbolic half-spaces containing $\Lambda(\Gamma)$. (A geodesic plane separates $H^{3}$ into two half-spaces.) Two points in $C(\Lambda)$ can be joined by a geodesic lying in $C(\Lambda)$; in particular, all lifts of the bounded trajectories, geodesics with initial and end points in $\Lambda(\Gamma)$, lie in $C(\Lambda) . C(\Lambda)$ is a compact three-dimensional domain, whose boundary consists of flat pieces, i.e., 


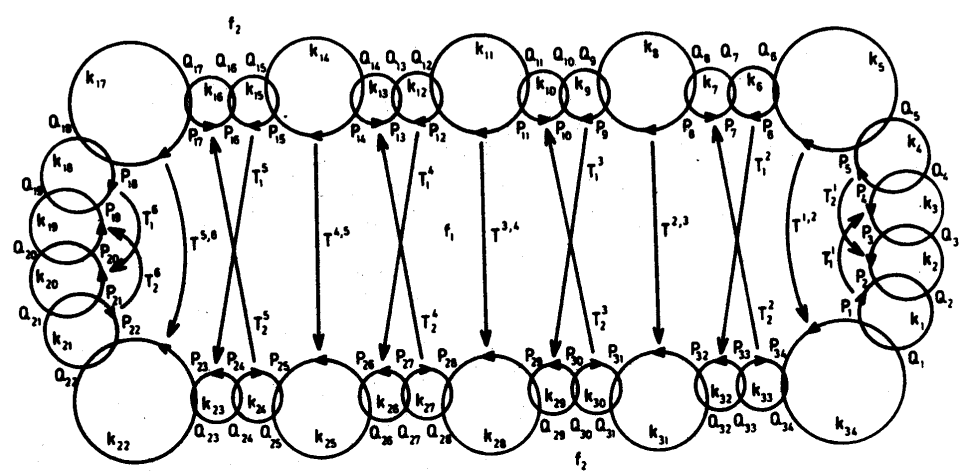

Fig. 5. As in Figure 2; placing hemispheres on the circles, we get the fundamental polyhedron: the space above the hemispheres and the two free polyhedral faces $f_{1}$ and $f_{2}$ (compactified). The identification of the faces on the hemispheres gives the manifold, a thickened surface of genus six.

domains that lie on geodesic planes, and bending lines, e.g., lines of intersection of geodesic planes or lines where lines of intersection accumulate. To visualize this, it is very helpful to draw first the two-dimensional Euclidean convex hull of the limit sets in the figures. An extensive treatise on $C(\Lambda)$ can be found in Epstein and Marden (1987). The intersection of $C(\Lambda)$ with $F$ gives via the face-identification of $F$ a realization of $C(\Lambda) \backslash \Gamma$.

Convexity properties are best studied in the Kleinian model of hyperbolic geometry $K^{3}$ (Ahlfors, 1981; Beardon, 1983; Thurston, 1978), in which the geodesics are straight lines and the concept of convexity is the same as in Euclidean space. $K^{3}$ is again a ball $|\mathbf{x}|<R$, endowed with

$$
d s^{2}=\frac{1}{1-|\mathbf{x}|^{2} / R^{2}} d \mathbf{x}^{2}+\frac{1}{R^{2}} \frac{1}{\left(1-|\mathbf{x}|^{2} / R^{2}\right)^{2}}(\mathbf{x} \cdot d \mathbf{x})^{2}
$$

The isometry $B^{3} \rightarrow K^{3}$ is given by $\mathbf{x} \rightarrow 2 \mathbf{x} /\left(1+|\mathbf{x}|^{2} / \mathbf{R}^{2}\right)^{2}$. It is the identity on the sphere at infinity $S_{\infty}$; a geodesic in $B^{3}$ is mapped onto a straight line that has the same intersection points with $S_{\infty}$. The geodesic planes bounding $F$ are Euclidean disks resting on the same base circles as the spherical caps in $B^{3}$. The bounded trajectories are now projections of straight lines with initial and end point in $\Lambda(\Gamma) . C(\Lambda)$ is the Euclidean convex hull.

We will now connect $\delta$ with the ground-state eigenvalue of the Schrödinger operator on the manifold, thus deriving an exact relation between a substantially quantum mechanical quantity, which is even in principle not accessible by semiclassical methods, with a geometrical quantity $\delta$, the Hausdorff dimension of $\Lambda(\Gamma)$. 
Fig. 6. As in Figure 3; the fibers are now surfaces of genus six corresponding to the identification pattern in Figures 5 and 12. Such tilings present simultaneous uniformizations of two Riemann surfaces (Bers, 1970), the boundary components of the manifold. $\delta=1.312$.

First we construct a Hausdorff cover of $\Lambda(\Gamma)$. We start with a ball $b\left(\mathbf{m}, r_{0}\right)$ in $F$ and apply $\Gamma$ to it; in every image of $F$ there lies therefore an image of $b$. The balls $\gamma(b)$ accumulate at the limit points. If $\gamma(b)$ lies close to $S_{\infty}, \gamma(b)$ will have a Euclidean radius

$$
r(\gamma) \approx \frac{r_{0}}{2}\left(1-\frac{|\gamma \mathbf{m}|^{2}}{R^{2}}\right)
$$

because of the conformal factor in the $B^{3}$-metric. Since the balls $\gamma(b)$ accumulate at $S_{\infty}$, their central projections from the origin onto $S_{\infty}$ provide Hausdorff covers (Sullivan, 1979). If the balls are close to $S_{\infty}$, the radii 
Fig. 7. The interior of the Jordan curve in Figure 6. Quasiconformal deformations of the unit disk give rise to quasi-self-similar shapes; see the caption of Figure 9.

$r_{c p}(\gamma)$ of these projections are approximately the same as those of the balls. The convergence abscissa of

$$
\sum_{\gamma \in \Gamma} r_{c p}(\gamma)^{s} \sim \sum_{\gamma \in \Gamma}\left(1-\frac{|\gamma \mathbf{m}|^{2}}{R^{2}}\right)^{s}
$$

( $s$ a complex parameter), is therefore the Hausdorff dimension $\delta$ of $\Lambda(\Gamma)$ ( $\sim$ means here only that the series have the same convergence abscissa). More rigorous arguments, with minimal covers, etc., are given in Sullivan (1979). The Hausdorff dimensions of the limit sets in Figures 3, 4, and 6-9 are calculated in Section 3.

The convergence abscissa of (2.4) also determines the ground state. If we represent the manifold as a fundamental polyhedron $F$ in $B^{3}$, Schrödinger's 
Fig. 8. Deformation spaces of hyperbolic manifolds are inhabited by many interesting species, their degree of differentiation depending on the genus of the fibers; here a tiling obtained by a deformation of the manifold in Figure 6. $\delta=1.338$.

eigenvalue equation reads

$$
-\frac{\hbar^{2}}{2 m}\left(\Delta_{B^{3}}+\frac{1}{R^{2}}\right) u=E u
$$

$u$ subject to periodic boundary conditions on the identified faces of $F$, i.e., $u\left(T_{i} \cdot\right)=u(\cdot)$ on the spherical caps identified by $T_{i}$. For the $T_{i}$ generate $\Gamma$ we have for all $\gamma \in \Gamma, u(\gamma \cdot)=u(\cdot)$ in $B^{3}$, i.e., $u$ automorphic with respect to $\Gamma$. In (2.5) $\Delta_{B^{3}}$ is the Laplace-Beltrami operator of $B^{3}$ and we have added 
Fig. 9. The interior component of Figure 8. Every small piece of the boundary curve $\Lambda(\Gamma)$ can be uniformly expanded to a standard size $r_{0}$, and then mapped back into $\Lambda(\Gamma)$ by a quasiisometry $Q$ (Ahlfors, 1966; McLaughlin, 1987; Sullivan, 1982), so that

$$
\frac{1}{K} \frac{r_{0}}{r}|x-y| \leq|Q(x)-Q(y)| \leq K \frac{r_{0}}{r}|x-y|
$$

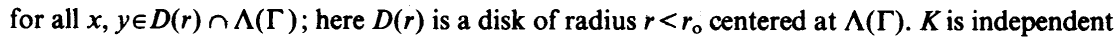
of $D(r)$, and can be taken as a measure for the deviation from self-similarity $(K=1)$.

$1 / R^{2}$ so that the continuous spectrum starts at zero. With the $B^{3}$-metric we calculate

$$
\Delta_{B^{3}}=\frac{1}{4}\left(1-\frac{r^{2}}{R^{2}}\right)^{2}\left[\Delta_{E^{3}}+\frac{1}{R^{2}} \frac{2}{1-r^{2} / R^{2}} r \frac{\partial}{\partial r}\right]
$$

$\Delta_{E^{3}}$ is the Euclidean Laplace operator.

The Green function $G_{E}^{\Gamma}(\mathbf{x}, \mathbf{y})$ is obtained as usual in periodic boundary value problems by periodizing the Green function $G_{E}^{B^{3}}(\mathbf{x}, \mathbf{y})$ of the covering space $B^{3}$. The Green function $G_{E}^{B^{3}}(\mathbf{x}, \mathbf{y})$ is defined (up to a normalization 
constant) by

$$
\left[\frac{\hbar^{2}}{2 m}\left(\Delta_{B^{3}}+\frac{1}{R^{2}}\right)+E\right] G_{E}^{B^{3}}(\mathbf{x}, \mathbf{y})=0 \quad \text { if } \quad \mathbf{x} \neq \mathbf{y}
$$

and by requiring a pole for $\mathbf{x}=\mathbf{y}$ and a decay as fast as possible for $|\mathbf{x}| \rightarrow R$. After introducing polar coordinates and variable separation in (2.7) (Elstrodt et al., 1983) one arrives at

$$
G_{E}^{B^{3}}(\mathbf{x}, \mathbf{0})=\frac{1}{8 \pi|\mathbf{x}|}\left(1+\frac{|\mathbf{x}|}{R}\right)^{-2(R / \hbar)(-2 m E)^{1 / 2}}\left(1-\frac{|\mathbf{x}|^{2}}{R^{2}}\right)^{1+(R / \hbar)(-2 m E)^{1 / 2}}
$$

For $R \rightarrow \infty$ we recover the Euclidean Green function; a rescaling of $|\mathbf{x}|$ is again necessary because of the factor $1 / 4$ in (2.6); cf. also (2.2).

The Green function $G_{E}^{\Gamma}(\mathbf{x}, \mathbf{y})$ of the manifold is given by

$$
G_{E}^{\Gamma}(\mathbf{x}, \mathbf{y})=\sum_{\gamma \in \Gamma} G_{E}^{B^{3}}(\gamma \mathbf{x}, \mathbf{y})
$$

It is an analytic function of the complex variable $E$; its poles and branch cuts determine the energy spectrum. The real positive axis is a branch cut corresponding to the continuous spectrum; $G_{E}^{\Gamma}(\mathbf{x}, \mathbf{y})$ is analytic in the halfplane $\operatorname{Re}(E)<0$ with the exception of a finite number of poles (bound states) on the negative real axis [cf. Elstrodt et al. (1983) and Lax and Phillips (1982) for a detailed discussion of these spectral properties].

The convergence abscissa of the series representation (2.9) of $G_{E}^{\Gamma}(\mathbf{x}, \mathbf{y})$ gives the first pole (Elstrodt et al., 1983; Patterson, 1975, 1976, 1987) and thus the ground-state energy. For $\Lambda(\Gamma)$ comprising the accumulation points of the orbit $\gamma \mathbf{x}, \gamma \in \Gamma$, we have $|\gamma \mathbf{x}| \rightarrow R$, and the first two factors in (2.8) are bounded and do not affect the convergence behavior of $G_{E}^{\Gamma}(\mathbf{x}, \mathbf{y})$. Thus, to determine the ground-state energy $E_{0}$ it is enough to determine the convergence abscissa of the Poincaré series

$$
h(\mathbf{x}, s)=\sum_{\gamma \in \Gamma}\left(1-\frac{|\gamma \mathbf{x}|^{2}}{R^{2}}\right)^{s}
$$

which was shown in (2.4) to be the Hausdorff dimension $\delta$ of $\Lambda(\Gamma)$. Thus, we have $\delta=1+(R / \hbar)\left(-2 m E_{0}\right)^{1 / 2}$, which is the formula given in the introduction.

\section{CHARACTERISTIC CURVES OF A TILING AND THE CALCULATION OF $\delta$}

Because we obtain $\Lambda(\Gamma)$ by tessellating the plane with $\Gamma$-images of $F$, it is intuitively clear that $\delta$ will depend on the number of tiles one needs to 
pp. 201-208 in attached file 


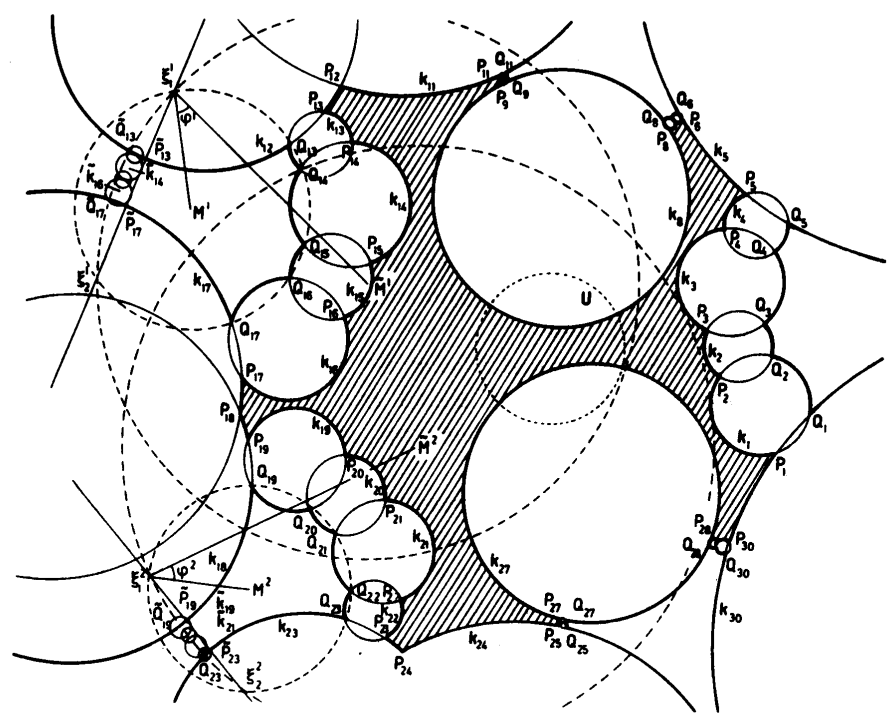

Fig. 13. As in Figure 12; realization of the identification pattern of Figure 2; the side-identifying transformations now satisfy (A4). The hatched domain is the center of Figure 3.

\section{ACKNOWLEDGMENTS}

The numerical calculations were done in the I. Prigogine Center for Statistical Mechanics at the University of Texas; I thank Lea Vetter and Paul Morris for assistance with the graphics. I thank P. Kinet for the drawings and I. Saverino for the typing of the manuscript. I also acknowledge discussions with D. Bessis and G. Mantica.

\section{REFERENCES}

Ahlfors, L. V. (1966). Lectures on Quasiconformal Mappings, Van Nostrand, New York.

Ahlfors, L. V. (1981). Möbius transformations in several dimensions, Lecture notes, University of Minnesota.

Apanasov, B. N. (1991). The Geometry of Discrete Groups and Uniformization, Reidel, Dordrecht.

Beardon, A. F. (1983). The Geometry of Discrete Groups, Springer, Berlin.

Bers, L. (1970). Annals of Mathematics, 91, 570-600.

Bessis, D., and Handy, C. (1986). International Journal of Quantum Chemistry, Quantum Chemistry Symposium, 20, 21-32.

Elstrodt, J., Grunewald, F., and Mennicke, J. (1983). Russian Mathematical Surveys, 38, 137 168.

Epstein, D. B. A., and Marden, A. (1987). Convex hulls in hyperbolic space, in Analytical and Geometrical Aspects of Hyperbolic Space, pp. 113-253, D. B. A. Epstein, ed., Cambridge University Press, Cambridge.

Ford, L. (1951). Automorphic Functions, Chelsea, New York. 
Hejhal, D. A. (1976). The Selberg Trace Formula for PSL(2, R), Vol. 1, Springer, Berlin. Hurt, N. E. (1983). Geometric Quantization in Action, Reidel, Dordrecht.

Krushkal, S. L. (1979). Quasiconformal Mappings and Riemann Surfaces, V. H. Winston \& Sons, Washington, D.C.

Krushkal, S. L., Apanasov, B. N., and Grusevskii, N. A. (1986). Kleinian Groups and Uniformization in Examples and Problems, American Mathematical Society, Providence, Rhode Island.

Lax, P. D., and Phillips, R. S. (1982). Journal of Functional Analysis, 46, 280-350.

Lehner, J. (1964). Discontinuous Groups and Automorphic Functions, American Mathematical Society, Providence, Rhode Island.

Lehto, O. (1987). Univalent Functions and Teichmüller Spaces, Springer, New York.

Marden, A. (1977). Geometrically finite Kleinian groups and their deformation spaces, in Discrete Groups and Automorphic Functions, pp. 259-293, W. J. Harvey, ed., Academic Press, London.

Maskit, B. (1974). Uniformizations of Riemann surfaces, in Contributions to Analysis, L. Ahlfors et al., eds., Academic Press, London.

McKean, H. P. (1972). Communications in Pure and Applied Mathematics, 25, 225-246.

McLaughlin, J. (1987). Proceedings of the American Mathematical Society, 100, 183-186.

Milnor, J. (1982). Bulletin of the American Mathematical Society (New Series), 6, 9-24.

Patterson, S. J. (1975). Monatshefte Math., 82, 297-315.

Patterson, S. J. (1976). Acta Mathematica, 136, 241-273.

Patterson, S. J. (1987). Lectures on measures on limit sets of Kleinian groups, in Analytical and Geometrical Aspects of Hyperbolic Space, pp. 281-323, D. B. A. Epstein, ed., Cambridge University Press, Cambridge.

Phillips, R. S., and Sarnak, P. (1985). Acta Mathematica, 155, 173-241.

Sullivan, D. (1979). Publ. Math. IHES, 50, 171-202.

Sullivan, D. (1982). Seminar on conformal and hyperbolic geometry, IHES Lecture Notes.

Sullivan, D. (1984). Acta Mathematica, 153, 259-277.

Thurston, W. (1978). The geometry and topology of 3-manifolds, Lecture Notes, Princeton University. Princeton, New Jersey.

Tomaschitz, R. (1989). Physica D, 34, 42-89.

Tomaschitz, R. (1991). Journal of Mathematical Physics, 32, 2571-2579.

Tomaschitz, R. (1992a). The topological structure of space-time and the microscopic dynamics, in Quantum Chaos - Quantum Measurement, P. Cvitanovic, ed., Kluwer Academic Publishers, Dordrecht.

Tomaschitz, R. (1992b). Chaotic dynamics in general relativity, to be published in Proceedings of a NATO Advanced Research Workshop on Chaotic Dynamics, July 11-20, 1991 Patras, Greece, T. Bountis, ed., NATO-ASI Series, Plenum, New York. 